\title{
REVIEW OF FSCM WITH BLOCKCHAIN AND BIG DATA INTEGRATION
}

\author{
Md. Ratul Amin \\ Universiti Kuala Lumpur \\ 1016, Jalan Sultan Ismail, Kuala Lumpur, Malaysia \\ md.ratul@s.unikl.edu.my \\ Muhammad Yasar Khan \\ Universiti Kuala Lumpur \\ 1016, Jalan Sultan Ismail, Kuala Lumpur, Malaysia \\ muhammadyasar@s.unikl.edu.my \\ *Megat F. Zuhairi \\ Universiti Kuala Lumpur \\ 1016, Jalan Sultan Ismail, Kuala Lumpur, Malaysia \\ megatfarez@unikl.edu.my \\ ${ }^{*}$ Corresponding author
}

\begin{abstract}
Distributed ledger technology is an immutable data storage and transparent system, which is a constituent component that empowers the FSCM (Food supply Chain Management). Due to the autonomous and immutable data feature, the scalability of blockchain technology is quite a challenge. A supply chain is a network that flows product from suppliers to the final consumer, which produces a high volume of data. In the blockchain, processing high volume of data is quite a significant issue and may affect business profit. To overcome the issue, Big Data and User Controllable Code for Smart Contracts (UCCSC) will be integrated with the blockchain-based FSCM system. Big data technology is typically used to analyze high volume of both structured and unstructured data, which is very difficult to process using habitual database and software techniques. The UCCSC integrates the blockchain and big data and manage the user in the FSCM system. This paper mainly discusses the integration of blockchain-based FSCM and Big Data, and the mechanism that supports such system.
\end{abstract}

Keywords: FSCM, Blockchain, Smart contracts, Big data, Data science

\section{Introduction}

Food Supply Chain Management (FSCM) is essential for both the food producer industries and consumers. Such system is designed to ensure transparency of food handling and process, leading to high quality of food production. Nonetheless, the Food Supply Chain Management (FSCM) is a lengthy process, which includes supplier, manufacturing, distributor, retailer and consumer. Each product is relayed through various stages of FSCM where information is recorded relevant to the product. In the end, every food product carries unique data along the FSCM process. The recorded data is vital because it provides feedback to the manufacturer as well as consumer about the source and the handling mechanism of the products. However, the challenges of the existing system are to sustain the integrity of information along the chain. Data tampering is one of the issues that severely affect the system [1][2]. Since 2009, immutable distributed ledger technology is gradually accepted as the transaction mechanism that could offer secure data processing with high scalability [3][4]. In general, blockchain is a chain of blocks, and the parent hash key is the key feature of the blockchain that makes the chain. The basic building blocks of the blockchain system includes parent hash key, timestamps, own hash key and transactions [5]. Nonetheless, the genesis block doesn't require the parent hash key. Secured block is provisioned by a couple of crypto-hash locks within the block, where the length of the hash key is set to minimum 256-bits [6]. The encryption mechanism can avoid unauthorized tampering with the data of the block. In addition, the timestamp is an essential tagging mechanism, which keeps the record of the time when the block is formed. The combination of block components binds the transaction data, which then allows the blockchain to keep the user record such as 
sender's data, receiver's data and users transaction e.g., amount of money transacted. Figure 1 shows the chain of block.

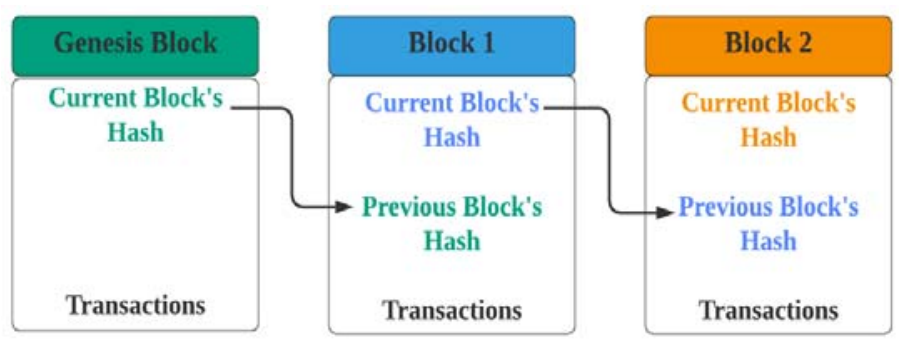

Figure 1: Chain of block

Figure 1 shows the chain of blocks, where the first block is called the genesis block that doesn't contain any previous block. The second block is generated by the signature of the genesis block, and it carries the key of the parent's block. Similarly, the third block is created by the signature of the second block and the previous block's hash is matched by the color in Figure 1. Such method is typically used to create a blockchain, which makes it secure and transparent than habitual technology.

The blockchain technology is classified by three types i.e., private, public, and consortium. On one hand, the private blockchain is open for everyone and public blockchain is the opposite of private blockchain. On the other hand, a consortium blockchain is a combination of the private and public blockchain. Indeed, the blockchainbased smart contract contains six-layer such as application layer, contract layer, incentive layer, consensus layer, network layer, and data layer [7]. Figure 2 represents the layer of blockchain.

- Data layer: All features are comprehensively connected in this layer i.e., timestamps, crypto hash, encryption algorithm, Merkle tree, and chain structure [8].

- Network layer: Peer to peer (P2P) networking, data transmission, and data verification are handled by the network layer [9]. Consensus layer: Proof-of-Work (PoW), Proof-of-Stake (PoS), and Delegate Proof of Stake (DPOS) are the consensus mechanisms that belong to the consensus layer [9].

- Incentive layer: A unique economic incentive and token allocation mainly maintain the blockchain network [10].

- Contract layer: It is a set of programming codes that works automatically without any additional manual intervention [11].

- Application layer: The blockchain technology has released different versions with additional features i.e., blockchain 1.0, blockchain 2.0, and blockchain 3.0 [12][7].

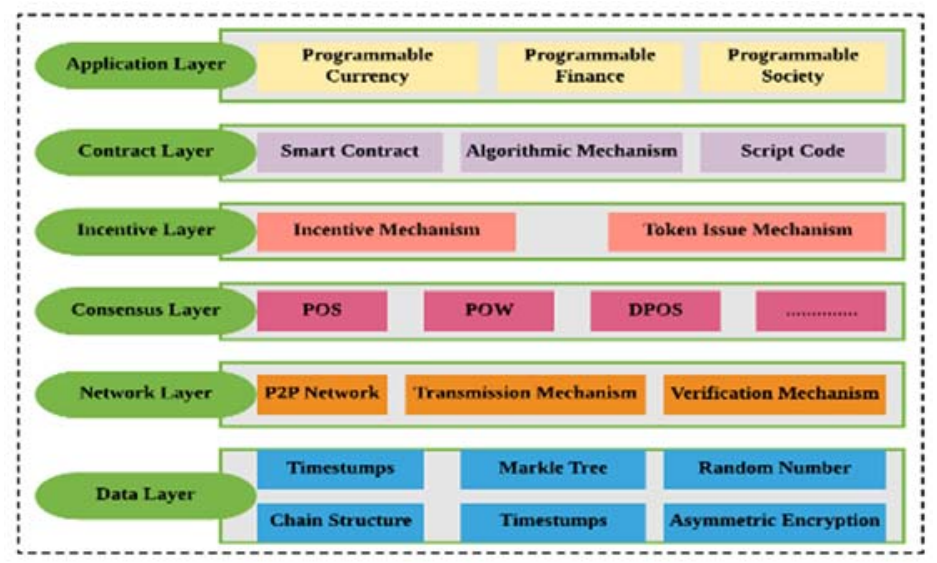

Figure 2: Layer of blockchain based smart Contracts

The blockchain technology can be used to complement Big Data where huge amount of data is transacted over the Internet. Big Data consortium of developers has released the guidelines for users which follows the $3 \mathrm{Vs}$ core landmark i.e., Velocity, Variety, and Volume [13]. These 3Vs are the typical identity of Big Data [14] but the extension of it may include few more categories i.e., Vision, Verification, Validation, and Value [15]. It is deemed 
that in recent years data has become the digital valuable asset such as structured or unstructured, public, or private, inside or outside the organizations. The data growth is nearly $50 \%$ per month and the expected total amount could be $800 \%$ in the next 5 years. It is expected by 2025 , the total sum of data will be around 50 zettabytes [16]. Hence, the blockchain and Big data technologies are essential to enterprises and consumers and may be able to significantly improves the business process. Subsequently, the FSCM process and data can be made transparent for every user along the supply chain, leading to better analysis to improve the business. The Food Supply Chain Management (FSCM) shown in Figure 3 is the use case of Blockchain with Big Data technology.

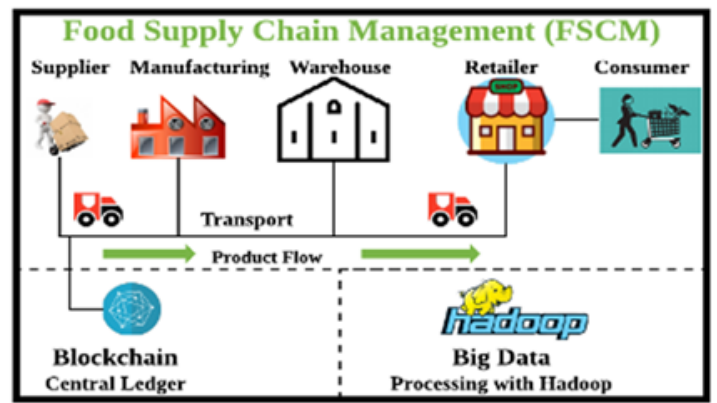

Figure 3: FSCM System with Blockchain \& Big Data

In the blockchain-based FSCM, to obstruct the execution of any malicious functions, the UCCSC is applied in the user code. Indeed, UCCSC is used to control the users of the application. It limits the number of executable operation codes (opcodes). In a computational code, the UCCSC's rules or codes will be set in a custom mapreduce Software Development Kit (SDK) specific to the UCCSC. By UCCSC, the values of the opcode will be tracked. Once the associated UCCSC values is exhausted, a gating system stops further opcodes in a computational code.

\section{Background}

\subsection{FSCM}

The FSCM is a method that creates a transparent connection between the users i.e., supplier, manufacturer, warehouse, retailer, and consumer [17]. Such technique offers businesses benefit by regulating the supply-chain network [18]. FSCM network is classified into different stages and substantial amount of time is needed to complete the whole process. In such circumstances, if the final product is damaged or of low quality, then identifying the cause of the fault can be very difficult [19][20]. Therefore, to ensure issues such as product faulty or to maintain quality, the movement of products must be tracked from origin to the end consumer and it is the fundamental part of FSCM [20].

\subsection{Smart Contract}

Blockchain is gradually being accepted as trustworthy technology, particularly when used with smart contracts [21]. Smart contracts can meet the whole business process independently without involving any third parties [22]. When a smart contract is deployed, the mechanism can operate autonomously [23]. Initially, the development of smart contracts i.e., a set of executable codes [23] that runs on blockchain technology, is driven by the event and state [24][25]. However, in the public blockchain, anonymous users may be part of the smart contracts that require high computation to transact data [26]. In addition to smart contract, the PoW is a particular consensus algorithm used in blockchain [27][28]. It is employed to ensure transacted data is validated by a group of users participating in the network. As such, if the smart contract requires high execution time or it contains an infinite loop, a severe delay may be incurred by the network. In this circumstance, Denial-of-Service (DoS) attacks might be fatal to the whole network [26]. Therefore, the development of smart contracts must keep in view of the delay and the consequences to the computation time.

\subsection{Hyperledger Fabric}

Hyperledger Fabric is an open-source, private and permissioned blockchain of Linux Foundation's project [29] [30]. The Membership Service Provider (MSP) is authorized to enroll the members of the fabric network. Hyperledger Fabric supports multiple MSPs along with ledger the data can be stored in multiple formats [31]. The 
Hyperledger Fabric's network permits to create the group among the participants and that group participants are able to make an individual ledger of transactions [26]. World state and transaction log are the main components of the ledger where the world state represents the state of the ledger at a given point of time and the transaction $\log$ is the update history for the world state. The smart contract is known as chain code in Hyperledger Fabric's platform that can be invoked by an external application if any required such as meet with the ledger. Particularly, it deals with the world state but disabled on transaction log [26]. To date, the Hyperledger Fabric's network is able to process 2000 transactions per second [32]. Such technology has become quite prominence and employed by more than 250 organizations such as IBM, Intel, Baidu, Huawei, ABN Amro, Accenture, and ANZ [32][33][34]35].

\subsection{Hadoop}

Hadoop is an open-source framework that enable a distributed large number of data set processing with clusters of computers [36][26]. It constructs two layers i.e., a storage component called Hadoop Distributed File System (HDFS), and a processing component using MapReduce programming model [37][38][39]. Hadoop has a Master Node to manage a set of Slave Nodes. The master-slave architecture of HDFS the Name Node or Master manages the file system metadata which stored in the slave or Data Nodes by creating the fixed size of the blocks. For ensuring the aliveness Data Nodes are continuously sends signals/heartbeats to the Name Node and mapping of blocks to the Data Nodes is determined by the Name Node. Data Nodes manage the read-write operations in the file system along with creation, deletion and replication of blocks, and it follows the instructions of the Name Node. HDFS's secondary Name Node stores checkpoints of the file system metadata. In the MapReduce, the Master Node is composed of a JobTracker and a NameNode. On the contrary, the Slave Nodes comprises of a TaskTracker and a DataNode. The JobTracker is accountable for scheduling tasks on TaskTrackers and monitoring their execution. Each TaskTracker has a configurable number of Map and Reduce slots, which limits the maximum number of simultaneous tasks in a node. At the execution time of tasks, a Slave Node sends heartbeats periodically to the Master Node, as a keepalive message. To that end, if the heartbeat is not received for a long period of time then the Slave Node is considered dead.

\subsection{User Controllable Code for Smart Contracts (UCCSC)}

According to the blockchain process, each smart contract can invoke itself or by using a function. The call stack is associated with the function for the reason of backward tracing of the calling sequence and is capable to keep $1024 \mathrm{~KB}$ at maximum [26]. The blockchain is growing with the rate of miner's capability in solving a reverse hash problem which increases the smart contracts function calls and may exceed the bounded limit, i.e., $1024 \mathrm{~KB}$. When stack limit exceeds, then exceptions will be generated.

\section{Related Work}

The improvement of FSCM is a pressing issue, which warrant research [40]. In October 2016, big retail industries i.e., Walmart and IBM have collaborated with Tsinghua University to explore the opportunities of blockchain in food products authentication and supply chain operation [41]. Walmart is one of the crucial IBM clients for testing the distributed ledger technology for FSCM. In another research work [40], Provenance Ltd. explores the value of blockchain in our society. The outcome of the work has identified a substantial reduction of food material waste during production and blockchain is able to keep good relationship between consumer and organization. In 2013, 50,000 tons of meat sold as beef, but later discovered as horse meat. To protect the consumers from the increasing risk of food fraud the Provenance Ltd. has developed a blockchain-based supply chain. The new approach ensures every product to be physically embedded with a digital identity that proves the authenticity and tracks the origin of the product. The current approach of supply chain process is acceptable, but it requires to implement an advance method to improve the consumer trustworthiness on the food product. Blockchain is the potential technology because of the data immutability feature. Data immutability and data share is the preventive strategy for food quality and security management, which is able to enhance consumer confidence in the food system. [42].

Since 2015, there is a growing interest of blockchain to be used in supply chain, on the account that the technology may still have significant challenges. To improve the efficiency of blockchain transaction, many research works exists to mitigate some of the issue [43]. Firstly, blockchain performance is inferior as compared to the traditional system counterpart. It is because when a transaction is executed, a blockchain employs similar computation resources as compared to the traditional database process. However, a distributed ledger system is 
required to process a single transaction three-fold, i.e., verification, consensus mechanisms, and redundancy. In addition, the node has to download the complete blockchain data for verification [43]. Secondly, blockchain the rate of growth of data is higher than the traditional system because each block piggybacks the previous hash key. Consequently, such approach may affect data processing time and data storage cost. In this circumstance, by enabling Big Data the employers will be able to fast process high-volume data and analysis them for business needs. As such, this paper proposed a novel approach Blockchain and Big Data integration that ensures the security and privacy of data to the data owner, as well as provides a user-controllable feature for third parties to apply their own code on the data.

\subsection{System Architecture}

To empower the FSCM, the core system blockchain technology integrates with Big data. The integration of Hyperledger Fabric and Hadoop is shown in Figure 4. The system architecture has three components i.e., a blockchain consortium, storage layer and, a computation layer, where data providers and data consumers are a particular part of it. The data providers are actually the owner of the data and it publishes the meta-data and also interacts with Big data resources for computation. The authorized data consumers are enabled to execute the code on the available data set and the smart contract is deployed with the consumer data. The chaincode in the Hyperledger Fabric preliminary observes the consumer code's malicious functions or infinite loops and reduces the complexity of computational. The storage layer of the HDFS is where data is stored, and the MapReduce framework is employed in the computation layer for distributed processing. The complete system's workflow is in 7 steps as shown by the Figure 5 .

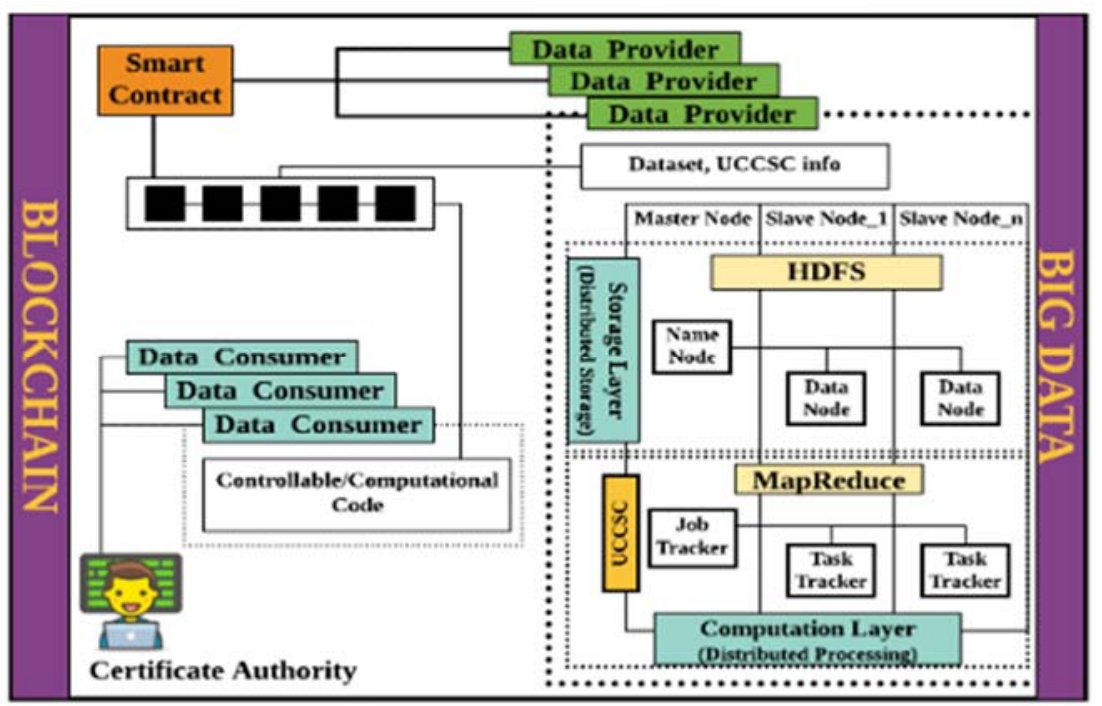

Figure 4: Hyperledger Fabric and Hadoop Integration Architecture

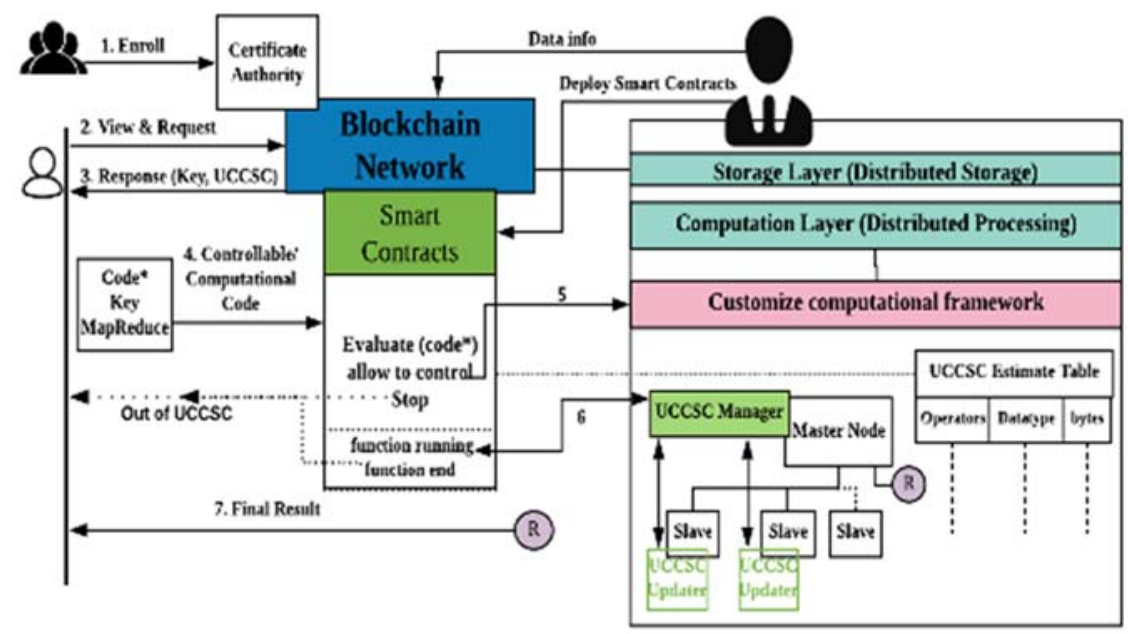

Figure 5: Integration Workflow of Blockchain and Big Data 
- Step 1: By registering into the system, the data consumer can be authorized in the blockchain consortium.

- Step 2: An authorized user can view the metadata as well provisioned with the computational power. A data consumer is able to estimate their needs based on metadata and their computational needs. As per network, the consumer could obtain the required UCCSCs from the blockchain consortium.

- Step 3: The data consumer will receive a response with the key to access the data, if the request is valid.

- Step 4: Based on the needs, the data consumer will provide the computational code, along with the provided key and acquired UCCSC. The submitted computational code must adhere to the custom map-reduce SDK. The smart contract then performs a preliminary check of the code that was deployed by the data provider. Subsequently, the smart contract evaluates whether the provided totem satisfies the computational needs of the provided code, which later the smart contract uses the information and a UCCSC estimator table to find the estimate of required UCCSC value. If the code is deemed valid by the smart contract, then it will allow the computation on the data.

- Step 5: Once the preliminary check passes, the actual computation will take place in a Customized Computational Framework (CCF). As the name indicates, it is customized and resembles the Hadoop architecture. Figure 5 also contains a UCCSC manager and a UCCSC updater. For each opcode execution, the slave node reports the performed opcode to the UCCSC updater in the slave node, which in returns send a response to the UCCSC manager in the master node. The UCCSC manager will then calculates the available balance based on the reports from all the updaters and sent the status back to all the updaters. When the updater receives the response from the manager, it then transmits a signal to the slave node for performing the next opcode. This operation repeats until the map or reduce function execution completes. Once a chain of map or reduce function is executed in the slave nodes, UCCSC updaters will update to the UCCSC manager. In this way, whenever the available UCCSC becomes end/zero, the UCCSC manager can immediately report an "Out of UCCSC" status to the blockchain and send a signal to master node to immediately stop the whole execution.

- Step 6: Once the execution of each map or reduce function is complete, the UCCSC manager sends the information regarding the used totem for that set of opcodes to the blockchain network as a transaction. The smart contract in the blockchain consortium will assess to see whether the consumer still has sufficient UCCSC for further computation. If it has enough balance, then control goes to the master node to perform further functions.

- Step 7: The data consumer will be able to see the result.

\section{Design and Method of Experiment}

The Hyperledger blockchain platform evaluated in this experiment is Hyperledger Fabric v1.2 and Hadoop v3.2.1. These experiments are conducted on a Lenovo Ideapad 320, and the machine specifications are Intel(R) Core (TM) i3-6006U CPU @ 2.00GHz, 8GB RAM, SSD 128GB, running Ubuntu 18.04. By varying the workload, performance will be evaluated to justify the proposed solution with 500 transactions and the metrics are execution time, latency, and throughput.

Hyperledger Caliper is the tool used to quantify blockchain performance. It allows users to test different blockchain platform to obtain the result. This paper used a modified version of Hyperledger Caliper. Hyperledger Caliper has some characteristics i.e., resource usage and transactions per second (TPS) and it generates HTML reports. The experiment conducted in this work is basically to calculate the transaction execution time.

The performance analysis of the system is shown in Figure 6 through Figure 8. The system's performance metrics are transaction time, latency time, and throughput time. The execution time of Hyperledger and Big Data system with varying number of transactions is $1,100,200,300,400,500$ in scale.

The integrated system's output is validated with other researcher's work i.e. transaction execution time, latency, and throughput. The Hyperledger performance measured by Dinh [43] and Suporn [44], where transaction execution time and latency time increases with high number of transactions. The throughput time, initially shown maximum transaction per second when the number of transactions increasing, while the throughput decreasing respectively. This integrated system's output also behave comparably, however the outputs are somewhat differing. The main difference between this paper experiment setup is the proposed scheme operates on the private application and the previously stated research works employed public application. 


\section{Transaction Execution Time}

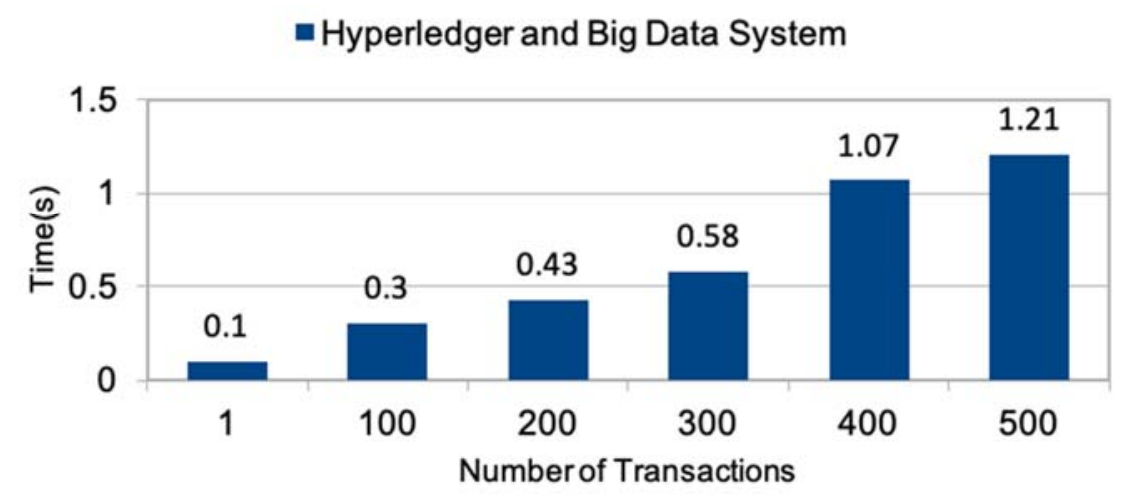

Figure 6: Transaction Execution Time

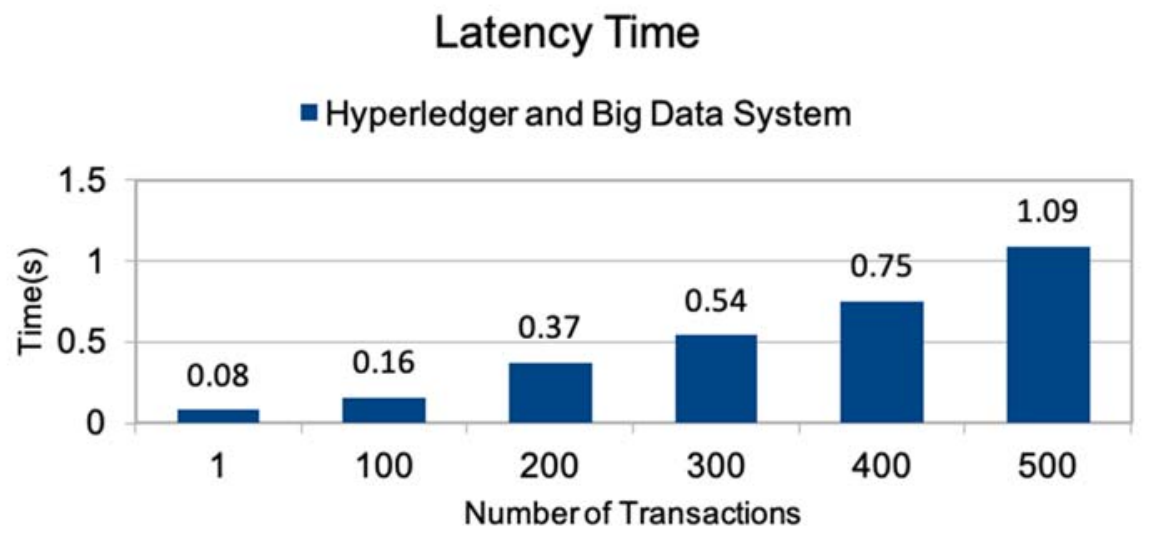

Figure 7: Latency Time

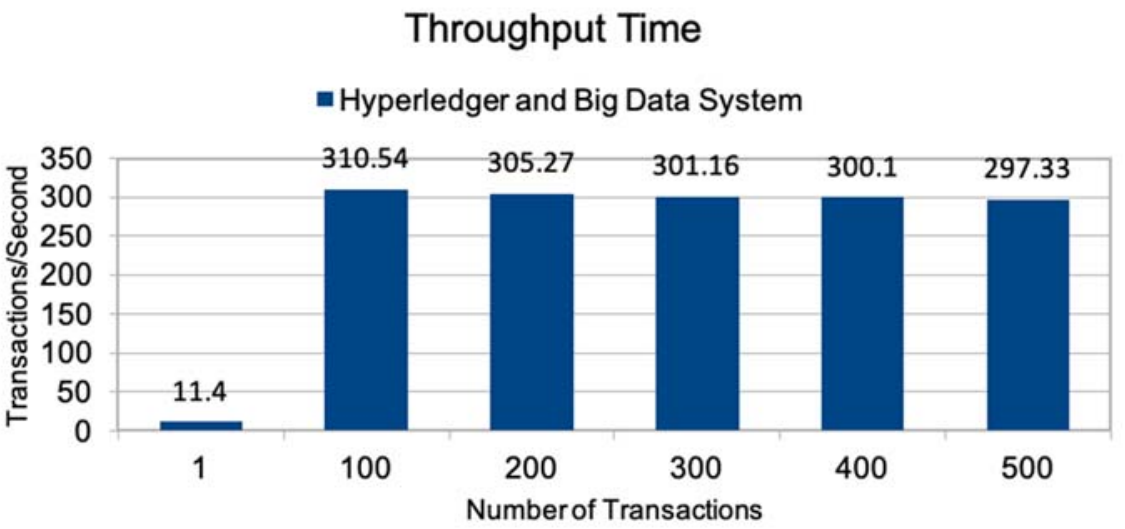

Figure 8: Throughput Time

\section{Result and Discussion}

This work investigates the execution time of varying the number of transactions. The execution time is shown by Figure 6, where bar graphs shows the transaction execution time of the integrated system. The transacted time is 0.10 second for first transaction. The transaction execution time increases with the number of transactions. At 500 number of transactions, the execution time of Hyperledger is 1.21 seconds. The comparison of latency in five sets of experiments is presented in Figure 7. The latency of integrated system for the first transaction is 0.08 second. When the number of transactions is increased from 100 to 200, the latency is high when compared with 
other data set. The throughput (transaction/second) of the integrated system shown by Figure 8 in unit of transaction per second. At the base point 100, the achieved maximum throughput is 310.69 transactions per second. At the base point 500 , the throughput is 298.33 transactions per second. Gradually, the throughput decreases with the number of increasing transactions.

\section{Conclusion}

In conclusion, the Blockchain technology on its own may not be sufficient to offer a complete control for user role within specific application. In this paper, the use case discussed in the FSCM. Within the supply-chain environment, users' interaction with the system may affect the process and even the product. Therefore, using Blockchain with Big data technology can offer a much-improved performance. Indeed, the FSCM is one of the domains that needs to use high-security technology because of concern on human health safety. Blockchain is one such vital technology that supports non-tampering data, and consequently it increases consumer confidence in the FSCM e.g., food products. Nonetheless, to run a fast business process, blockchain may has a slight downside because it produced a huge amount of data that effect on the business process. The integration between Big data with blockchain technology may somewhat alleviate the issue and process data rapidly and with provision of control to users for a particular role.

\section{References}

[1] C. Xia, Yan Sun, Hong Luo, "Secured Data Storage Scheme based on Block Chain for Agricultural Products Tracking", 3rd International Conference on Big Data Computing and Communications, (2017) pp. 45 - 50, 2017.

[2] T. Bocek, Bruno B. Rodrigues, Tim Strasser, Burkhard Stiller, "Blockchains Everywhere - A Use-case of Blockchains in the Pharma Supply-Chain”, IFIP/IEEE Symposium on Integrated Network and Service Management (IM), 2017.

[3] Zibin Zheng, Shaoan Xie, Hongning Dai, Xiangping Chen, and Huaimin Wang, "An Overview of Blockchain Technology: Architecture, Consensus, and Future Trends", IEEE 6th International Congress on Big Data, 2017.

[4] Michael Crosby, Nachiappan, Pradan Pattanayak, Sanjeev Verma, Vignesh Kalyanaraman, "BlockChain Technology: Beyond Bitcoin", 2016.

[5] Ali Dorri, Salil S. Kanhere, Raja Jurdak, Parveen Gauravaram, "Blockchain for IoT security and privacy: The case study of a smart home", IEEE International Conference on Pervasive Computing and Communication Workshops (PerCom Workshops), 2017.

[6] Mohammad Badrul Hossain, Sadman Sakib Akash, Md. Asraful Haque, "coinBD: An Enhanced Version of Proof of Work with Less Computational Power", 2018.

[7] Zheng Che, Yu Wang, Juanjuan Zhao, Yan Qiang, Yue Ma and Jihua Liu, “A Distributed Energy Trading Authentication Mechanism Based on a Consortium Blockchain", 2019.

[8] Qalab E Abbas, Jang Sung-Bong, "A Survey of Blockchain and Its Applications”, International Conference on Artificial Intelligence in information and Communication (ICAIIC), 2019.

[9] Ali Vatankhah BARENJI, Hanyang GUO, Zonggui TIAN, Zhi LI, W.M. WAN Gaand George Q. HUANG, "Blockchain-Based Cloud Manufacturing: Decentralization", 2019.

[10] Haojuan Mei, Zhen Gao, Zhaohui Guo, Ming Zhao, and Jinsheng Yang, "Storage Mechanism Optimization in Blockchain System Based on Residual Number System”, 2019.

[11] Zhiliang Deng,Yongjun Ren,Yepeng Liu,Xiang Yin, Zixuan Shen, and Hye-Jin Kim, "Blockchain-Based Trusted Electronic RecordsPreservation in Cloud Storage", 2019.

[12] Zixiao Xu, Dechang Yang, and Weilin Li, "Microgrid Group Trading Model and Solving Algorithm Based on Blockchain", 2019.

[13] Muhammad Younas, "Research challenges of big data", Service Oriented Computing and Applications, pp.105-107, 2019.

[14] B. Chidambararajan, M. Senthil Kumar, S.K.Susee, "Big Data Privacy and Security Challenges in Industries", International Research Journal of Engineering and Technology (IRJET), 2019.

[15] Fatima-Zahra Benjelloun, Ayoub Ait Lahcen, "Big Data Security: Challenges, Recommendations and Solutions", 2015.

[16] Mundial FE, "Big data, big impact: new possibilities for international development, Switzerland", 2016.

[17] Mentzer, J. T., Dewitt, W., Keebler, J. S., Min, S., Nix, N. W., Smith, C. D., \& Zacharia, Z. G., "Defining supply chain management", Journal of Business Logistics, pp.1-25, 2001.

[18] Shoue Chen, Sandrayee Brahma, Jonathon Mackay, Changyong Cao, Bahar Aliakbarian, "The role of smart packaging system in food supply chain", 2020.

[19] Galvez, Juan F., J. C. Mejuto, and J. Simal-Gandara. "Future challenges on the use of blockchain for food traceability analysis." TrAC Trends in Analytical Chemistry 107, pp. 222-232, 2018.

[20] Affaf Shahid, Umair Sarfraz, Muhammad Waseem Malik, Muhammad Sohaib Iftikhar, Abid Jamal, and Nadeem Javaid, "Blockchainbased Reputation System in Agri-Food Supply Chain”, 2020.

[21] Zibin Zheng,Shaoan Xie, Hong-Ning Dai, Weili Chen, Xiangping Chen, Jian Weng, Muhammad Imran, "An Overview on Smart Contracts: Challenges, Advances and Platforms", 2020.

[22] Tonglai Liu, Jigang Wu, Long Chen, Yalan Wu, And Yinan Li, "Smart Contract-Based Long-Term Auction forMobile Blockchain Computation Offloading", 2020.

[23] N. Szabo, "Smart contracts: Building blocks for digital markets", Extropy, J. Transhumanist Thought, vol. 18 , pp. 2, 1996.

[24] P. Wang, J. Meng, J. Chen, T. Liu, Y. Zhan, W.-T. Tsai, and Z. Jin, "SmartAC negotiation for adaptive QoS-aware service composition" IEEE Trans.Parallel Distrib. Syst., vol. 30, pp. 1403-1420, 2019.

[25] Z. Li, H. Guo, W. M. Wang, Y. Guan, A. V. Barenji, G. Q. Huang,K. S. McFall, and X. Chen, "A blockchain and autoML approach for openand automated customer service, ” IEEE Trans Ind. Informat., vol. 15, pp. 3642-3651, 2019.

[26] Dhanya Therese Jose, Antorweep Chakravorty, Chunming Rong, "TOTEM: Token for controlled computation: Integrating Blockchain with Big Data", 2019.

[27] Satoshi Nakamoto, "Bitcoin: A peer-to-peer electronic cash system", bitcoin.org, 2009.

[28] L. Guo, H. Xie, Y. Li, "Data Encryption based Blockchain and Privacy Preserving Mechanisms towards Big Data", J. Vis. Commun. Image R., 2019.

[29] Androulaki, E., Barger, A., Bortnikov, V., Cachin, C., Christidis, K., De Caro, A., ... \& Muralidharan, S., "Hyperledger fabric: a distributed operating system for permissioned blockchains", Thirteenth EuroSys Conference, pp. 30, 2018,

[30] Parth Thakkar and Senthil Nathan, "Scaling Hyperledger Fabric Using Pipelined Execution and Sparse Peers”, 2020. 
[31] Nicolae Berendea, Hugues Mercier, Emanuel Onica, and Etienne Riviere, "Fair and Efficient Gossip in Hyperledger Fabric", 2020.

[32] Jian Chen a, Zhihan Lv a, Houbing Song b, "Design of personnel big data management system based on blockchain", Future Generation Computer Systems 101, pp. 1122-1129, 2019

[33] P. Thakkar, S. Nathan, B. Viswanathan, "Performance benchmarking and optimizing hyperledger fabric blockchain platform", in: 2018 IEEE 26th International Symposium on Modeling, Analysis, and Simulation of Computer and Telecommunication Systems (MASCOTS), IEEE, pp. 264-276, 2018.

[34] A. Baliga, N. Solanki, S. Verekar, A. Pednekar, P. Kamat, S. Chatterjee, "Performance characterization of hyperledger fabric", in: 2018 Crypto Valley Conference on Blockchain Technology (CVCBT), IEEE, pp. 65-74, 2018.

[35] Dean, J., \& Ghemawat, S., "MapReduce: A Flexible Data Processing Tool", Communications of the ACM, 53(1), pp. 72-77, 2010

[36] Borthakur, D. "The Hadoop Distributed File System: Architecture and Design", Hadoop Project Website, 11, pp. 21, 2007.

[37] Ankit Shah, Mamta C. Padole, "Saksham Model" Performance Improvisation Using Node Capability Evaluation in Apache Hadoop", 2020.

[38] Buterin, V., "A next-generation smart contract and decentralized application platform", White paper, 2014.

[39] Y. Wang, Jeong Hugh Han and Paul Beynon-Davies, "Understanding blockchain technology for future supply chains: a systematic literature review and research agenda", Supply Chain Management: An International Journal, pp. 62-84, 2018

[40] H. Sternberg, G. Baruffaldi, "Chains in Chains - Logic and Challenges of Blockchains in Supply Chains", Hawaii International Conference on System Sciences, 2018.

[41] D. Tse, B. Zhang, Y. Yang, C. Cheng, H. Mu, "Blockchain Application in Food Supply Information Security", IEEE International Conference on Industrial Engineering and Engineering Management (IEEM), pp. 1357 - 1361, 2017.

[42] Md. Ratul Amin, Megat F. Zuhairi and Md. Nazmus Saadat, "Enhanced Blockchain Transaction: A Case of Food Supply Chain Management", Journal of Engineering and Applied Sciences 15 (1), pp. 99-106, 2020.

[43] T. T. A. Dinh, J. Wang, G. Chen, R. Liu, B. C. Ooi, and K.-L. Tan, "Blockbench: A framework for analyzing private blockchains", 2017.

[44] Suporn Pongnumkul, Chaiyaphum Siripanpornchana, and Suttipong Thajchayapong, "Performance Analysis of Private BlockchainPlatforms in Varying Workloads", 2017. 